\title{
Nanoscale optomechanical sensors: split-beam photonic crystal nanocavities
}

\author{
Aaron Hryciw ${ }^{\dagger}$, Marcelo $\mathrm{Wu}^{* \dagger}$, Behzad Khanaliloo*†, Christopher Healey ${ }^{* \dagger}$, Paul E. Barclay*† \\ *Institute For Quantum Science And Technology \\ University of Calgary, AB, Canada, T2N1N4 \\ Email: see http://www.iqst.ca/people/ \\ ${ }^{\dagger}$ NRC-National Institute For Nanotechnology \\ 11421 Saskatchewan Drive NW, Edmonton, AB, Canada, T6G 2M9
}

\begin{abstract}
Optomechanical nanocavities allow nanomechanical resonances to be measured optically with high sensitivity. We have created a new type of photonic crystal nanocavity optomechanical sensor optimized for detecting sources of torque and other forces which can deflect nanoscale cantilevers. This nanocavity consists of two precisely engineered photonic Bragg mirrors patterned in silicon cantilevers and separated by a $50-100 \mathbf{n m}$ wide gap. Simulations of the optical and mechanical modes predict that mechanical displacements of the sub-picogram cantilevers will shift the optical nanocavity resonance frequency at a rate exceeding $20 \mathrm{GHz} / \mathrm{nm}$, and that the nanocavity optical mode may have a quality factor $Q_{o}>10^{6}$ in optimized devices.
\end{abstract}

\section{INTRODUCTION}

In recent years, optomechanical nanocavities have enabled detection of sub-pm motion of micro- and nano-scale objects, allowing the realization of force sensors with record sensitivity [1], [2], [3] and studies of the quantum properties of mechanical objects [4]. These devices consist of nanostructures which support optical and mechanical resonances which overlap in space. The optical nanocavity can be formed from dielectric materials patterned with a variety of geometries, including photonic crystal and whispering gallery mode optical resonators, and typically support optical modes with frequencies in the $200-600 \mathrm{THz}$ range (wavelengths from visible to near-IR), high optical quality factor, $Q_{o}$, and wavelength-scale optical mode volumes. Mechanical resonances of these nanocavities, or of mechanical structures placed in the optical nanocavity near-field, can be engineered to have frequencies ranging from $\mathrm{kHz}-\mathrm{GHz}$, and effective mass below a fg. Here we analyze a new type of optomechanical nanocavity called a "splitbeam" photonic crystal nanocavity, which combines strong optomechanical coupling with high sensitivity detection of a variety of torsional and cantilever mechanical modes.

\section{DESIGN RATIONALE}

Motion of mechanical resonances within optomechanical nanocavities is readout by monitoring changes in the optical response of the nanocavity to which they are coupled. Transduction of mechanical motion onto a change in the optical nanocavity response results from modification of the dielectric environment of the optical nanocavity field as a function of mechanical resonator position. This transduction can be understood by considering the frequency dependent
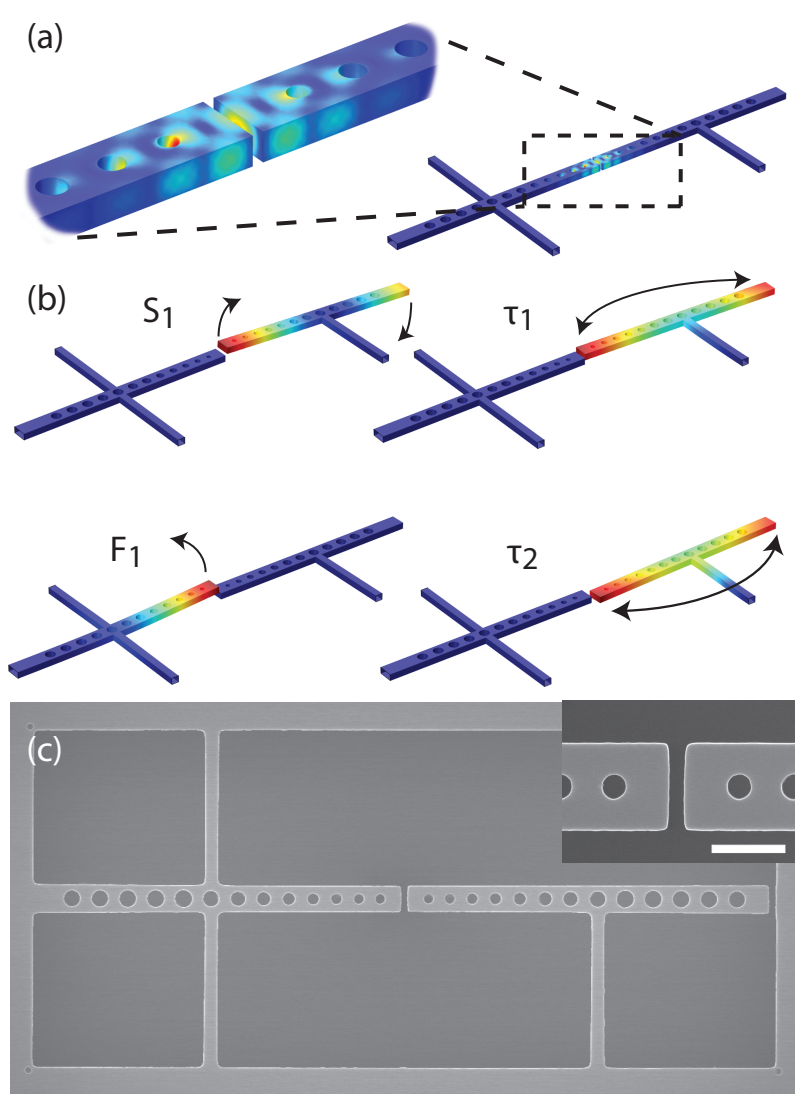

Fig. 1. a) Finite element (FEM) simulation of the TE-like optical field inside the nanocavity. Warmer colors correspond to stronger electric field intensity. b) Mechanical mode shapes as determined by FEM simulations: out-of-plane "see saw" $\mathrm{S}_{1}=5.12 \mathrm{MHz}$, in-plane first torsional $\tau_{1}=5.28 \mathrm{MHz}$, out-ofplane flexural $\mathrm{F}_{1}=9.89 \mathrm{MHz}$ and in-plane second torsional $\tau_{2}=18.72 \mathrm{MHz}$. $\tau_{1}$ flexes on the side of the support while $\tau_{2}$ has its pivot point in the halfnanobeam. The support is $3 \mu \mathrm{m}$ long and $250 \mathrm{~nm}$ wide. Warmer colors signify larger displacement which was exaggerated to demonstrate mechanical motion. c) a) Scanning electron micrograph of the fabricated split-beam nanocavity. The inset shows the $80 \mathrm{~nm}$ split with the scale bar indicating $500 \mathrm{~nm}$.

transmission of an optical waveguide coupled to a nanocavity:

$$
T(\omega)=S\left|1-\frac{\gamma_{e}}{i\left(\omega-\omega_{o}\right)+\gamma_{t} / 2}\right|^{2}
$$

where $S$ is the power input to the waveguide, $\gamma_{e}$ is the rate of optical coupling between the optical waveguide and 
nanocavity, $\gamma_{t}$ is the total energy decay rate of the nanocavity, and $\omega_{o}$ is the optical nanocavity resonance frequency. In optomechanical nanocavities, $\omega_{o}, \gamma_{e}$ and $\gamma_{t}$ may each depend on the displacement, $x$, of a mechanical resonator interacting with the optical nanocavity field. In general, $x$ describes the displacement amplitude of a specific mode of the mechanical resonator. To quantify the optomechanical coupling, it is necessary to calculate the rate of change in optical nanocavity parameters as a function of mechanical displacement. For example, in the case of purely dispersive coupling $\left(\omega_{o}=\right.$ $\omega_{o}(x), \gamma_{e, t}$ independent of $\left.x\right)$, a small displacement $d x$ of the mechanical resonator will induce the following change in waveguide transmitted power:

$$
d T=\frac{d T}{d \omega_{o}} \frac{d \omega_{o}}{d x} d x=\frac{d T}{d \omega_{o}} g_{\mathrm{om}} d x
$$

where $g_{\mathrm{om}}$ is the optomechanical coupling coefficient. The above equation assumes that the mechanical frequency is small compared to the nanocavity photon decay rate, $\omega_{m} \ll \gamma_{t}$, so that backaction related to the delay in nanocavity response to mechanical displacement can be neglected. In the more general case, coupling between the mechanical and optical equations of motion must be analyzed [5]. In order to maximize $d T / d x$, it is necessary to simultaneously maximize the optomechanical couping, $g_{\mathrm{om}}$, by ensuring that the optical and mechanical modes have strong spatial overlap and appropriate symmetries [6], and minimize $\gamma_{t}$ by creating an optical nanocavity with high optical quality factor $Q_{o}=$ omega $a_{o} / \gamma_{t}$. If the device is to be used as a force sensor, it is also necessary to consider the mechanical susceptibility of the mechanical resonator, which describes the coupling between $d x$ and an external force. Typically, it is desirable to minimize the mass of the resonator, maximize its mechanical quality factor $Q_{m}$, and engineer $\omega_{m}$ to be resonant with the driving force.

\section{PHOTONIC CRYSTAL SPLIT-BEAM OPTOMECHANICAL NANOCAVITY DESIGN}

With the above criteria in mind, we have recently designed a novel optomechanical nanocavity, consisting of two precisely engineered photonic Bragg mirrors patterned in silicon cantilevers and separated by a $50-100 \mathrm{~nm}$ wide gap. This optomechanical nanocavity is called a photonic crystal "splitbeam" nanocavity, and is illustrated in Fig. 1. Despite the large perturbation introduced by the gap in the center of the nanocavity to the refractive index profile of the nanobeam, a high $Q_{o}$ optical mode can be supported by careful design of the hole dimensions through band-gap matching [7]. In this design, an optical potential is smoothly varied from the outer "Bragg mirror" region to the central gap region, allowing a localized optical mode to be tightly confined to the center of the nanocavity. The spatially varying optical potential can be calculated from the bandedge of each unit cell of the nanocavity, assuming that the unit cell is repeated periodically. In the design shown here, $Q_{o}>10^{4}$, while in further optimized designs, $Q_{o}>10^{6}$ is possible [7]. The optical field distribution of the high $Q_{o}$ mode for the device considered here is shown in Fig. 1(a), and is predicted to exist at a wavelength $\lambda_{o} \sim 1550 \mathrm{~nm}=2 \pi c / \omega_{o}$. As discussed below, small changes in the gap width dramatically shift the optical frequency of this mode, resulting in strong optomechanical coupling.
The central gap, combined with a supporting structure in which one of the nanobeams is only suspended by a single support connecting it to the surrounding device layer, opens up mechanical degrees of freedom such as those shown in Fig. 1(b). This mechanical design allows the device to be efficiently actuated by external sources of torque, and the mechanical resonant frequencies can be easily tailored for an application of interest by modifying the dimensions of the supporting structure. For the design presented here, assuming the source of torsional motion produces a torque vector pointing out of the plane of the device, this motion will couple favorably to inplane mechanical modes such as the torsional modes at $5 \mathrm{MHz}$ $\left(\tau_{1}\right)$ and $19 \mathrm{MHz}\left(\tau_{2}\right)$. Using perturbation theory [6], these inplane modes are predicted to have $g_{\text {om }} \sim 20 \mathrm{GHz} / \mathrm{nm}$, allowing efficient optomechanical detection. Due to the symmetry of the optical field, vertical cantilever modes such as $S_{1}$ and $F_{1}$ have zero optomechanical coupling. However, the presence of an optical fiber in the nanocavity near-field can break this symmetry and induce a significant $g_{\text {om }}$, allowing observation of optomechanical coupling to these modes even in the limit of thermal Brownian motion. An example of a fabricated device is shown in Fig. 1(c). Measurements of the optomechanical properties of this device is on-going, and will be presented in future work.

\section{CONCLUSION}

We have designed and fabricated a new type of optomechanical nanocavity which is expected to be a sensitive detector of external force and torque. The optical and mechanical modes of this structure overlap spatially, resulting in large optomechanical coupling. Small displacements of the mechanical resonator actuate large shifts in the optical nanocavity frequency, which can be sensitively measured in the nanocavity $Q_{o}$ is sufficiently high.

\section{ACKNOWLEDGMENT}

The authors would like to thank Mark Freeman and John Davis for helpful discussions. This works is supported by NSERC, NRC, CFI and AITF.

\section{REFERENCES}

[1] Y. Liu, H. Miao, V. Aksyuk, and K. Srinivasan, "Wide cantilever stiffness range cavity optomechanical sensors for atomic force microscopy," Opt. Express, vol. 20, no. 16, pp. 18 268-18 280, 2012.

[2] E. Gavartin, P. Verlot, and T. Kippenberg, "A hybrid on-chip optomechanical transducer for ultrasensitive force measurements," Nat. Nano., vol. 7, no. 8, pp. 509-514, 2012.

[3] A. G. Krause, M. Winger, T. D. Blasius, W. Lin, and O. Painter, "A high-resolution microchip optomechanical accelerometer," Nat. Photon., vol. 6, no. 11, pp. 768-772, 2012.

[4] J. Chan, T. P. M. Alegre, A. H. Safavi-Naeini, J. T. Hill, A. Krause, S. Groblacher, M. Aspelmeyer, and O. Painter, "Laser cooling of a nanomechanical oscillator into its quantum ground state," Nature, vol. 478, pp. 89-92, 2011.

[5] T. Kippenberg and K. Vahala, "Cavity opto-mechanics," Opt. Express, vol. 15, no. 25, pp. $17172-17205,2007$.

[6] M. Eichenfield, J. Chan, A. Safavi-Naeini, K. Vahala, and O. Painter, "Modeling dispersive coupling and losses of localized optical and mechanical modes in optomechanical crystals," Opt. Express, vol. 17, no. 22, pp. 20078-20 098, 2009.

[7] A. Hryciw and P. E. Barclay, "Optical design of split-beam photonic crystal nanocavities," arXiv:1301.0367, 2013. 